\title{
T951
}

\section{ALICE Electromagnetic Calorimeter Prototype Test}

\author{
September, 2005
}


$\begin{array}{ll}\text { INTRODUCTION } & 3\end{array}$

I. PERSONNEL AND INSTITUTIONS:

II. EXPERIMENTAL AREA, BEAMS AND SCHEDULE CONSIDERATIONS 5

III. $\quad$ RESPONSIBILITIES BY INSTITUTION - NON FERMILAB

IV. $\quad$ RESPONSIBILITIES BY INSTITUTION - FERMILAB

4.1 Fermilab Accelerator Division 7

4.2 Fermilab Particle Physics Division 7

4.3 Fermilab Computing Physics Division 8

$\begin{array}{lll}4.4 & \text { Fermilab ES\&H Section } & 8\end{array}$

$\begin{array}{lll}\text { V. } & \text { SUMMMARY OF COSTS } & 8\end{array}$

$\begin{array}{lll}\text { VI. } & \text { SPECIAL CONSIDERATIONS } & 9\end{array}$

$\begin{array}{ll}\text { SIGNATURES } & 10\end{array}$

APPENDIX I - APPENDIX II - PREP EQUIPMENT POOL NEEDS 11

APPENDIX II - HAZARD IDENTIFICATION CHECKLIST 12 


\section{INTRODUCTION}

This Memorandum of Understanding between the Test Beam collaborators and Fermilab is for the use of beam time at Fermilab during the Fall, 2005 Meson Test Beam Run. The experimenters plan to measure the energy, position, and time resolution of prototype modules of a large electromagnetic calorimeter proposed to be installed in the ALICE experiment at the LHC. The ALICE experiment is one of the three large approved LHC experiments, with ALICE placing special emphasis on the LHC heavy-ion program. The large electromagnetic calorimeter (EMCal) is a US initiative that is endorsed by the ALICE collaboration and is currently in the early stages of review by the Nuclear Physics Division of the DOE. The installation in the test beam at FNAL and test beam measurements will be carried out by the US members of the ALICE collaboration (ALICE-USA).

The overall design of the ALICE EMCal is heavily influenced by its location within the ALICE L3 magnet. The EMCal is to be located inside the large room temperature magnet within a cylindrical integration volume approximately $112 \mathrm{~cm}$ deep, by $5.6 \mathrm{~m}$ in length, sandwiched between the ALICE TPC space frame and the L3 magnet coils. The chosen technology is a layered $\mathrm{Pb}$-scintillator sampling calorimeter with a longitudinal pitch of $1.6 \mathrm{~mm} \mathrm{~Pb}$ and $1.6 \mathrm{~mm}$ scintillator. The full detector spans $\eta=-0.7$ to $\eta=0.7$ with an azimuthal acceptance of $\Delta \phi=$ $120^{\circ}$. The EMCal readout is of a "Shish-Kabob" type similar to the PHENIX Pb-scintillator sampling calorimeter ${ }^{1}$ in which the scintillation light is collected via wavelength shifting fibers running through the $\mathrm{Pb}$-scintillator tiles perpendicular to the front surface. The detector is segmented into $\sim 14000$ towers.

\begin{tabular}{|l|l|}
\hline Quantity & Value \\
\hline Sampling Ratio, $\mathrm{d}_{\mathrm{Pb}} / \mathrm{d}_{\mathrm{Sc}}$ & $1.6 \mathrm{~mm} \mathrm{~Pb} / 1.6 \mathrm{~mm}$ Scintillator \\
\hline Sampling Fraction, $\mathrm{f}_{\mathrm{s}}=\Delta \mathrm{E}_{\mathrm{Sd}} \mathrm{d} \Delta \mathrm{E}_{\mathrm{Pb}}$ & 0.0811 (including all materials) \\
\hline Total depth & $24.8 \mathrm{~cm}$ \\
\hline Number of $\mathrm{Pb} / \mathrm{Sc}$ cells & 78 \\
\hline Number of Radiation Lengths & 22.3 \\
\hline Module Size & $12.7 \times 12.6 \times 31 \mathrm{~cm}^{3}$ \\
\hline Tower Size (at eta=0) & $\Delta \phi \times \Delta \eta=0.015 \times 0.015$ \\
\hline Number of Towers & 13,824 \\
\hline Number of Modules & $12 \times 12 \times 24=3456$ \\
\hline Number of Supermodules & 12 \\
\hline Weight of Supermodule & $\sim 9.6$ tons \\
\hline Total Coverage & $\Delta \phi=120^{\circ},-0.7<\eta<0.7$ \\
\hline
\end{tabular}

Table 1. The ALICE EMCal characteristics.

The basic structural units of the calorimeter are supermodules, each subtending approximately $\sim 20^{\circ}$ in $\Delta \phi$ and 0.7 units in $\Delta \eta$. Supermodules are assembled from individual modules. The modules are further segmented into $2 \times 2$ individually read out towers. The fibers from an individual tower are grouped together to form readout tower bundles. These are each optically

${ }^{1}$ L. Aphecetche et al. (PHENIX), Nucl. Instrum. Meth. A499, 521 (2003). 
coupled to an avalanche photodiode (APD) via a short lightguide to provide some spatial optical mixing and to match the fiber bundle to the APD. The module assembly is indicated in Figure 1. The supermodules weigh about 9.6 tons and are the basic units handled during installation. Each supermodule is roughly $145 \mathrm{~cm}$ wide at the front surface by $350 \mathrm{~cm}$ long with an active depth of $24.5 \mathrm{~cm}$ (at $\eta=0$ ) plus an additional $6.6 \mathrm{~cm}$ of depth in structural plates. The physical characteristics of the ALICE EMCal are summarized in Table 1.

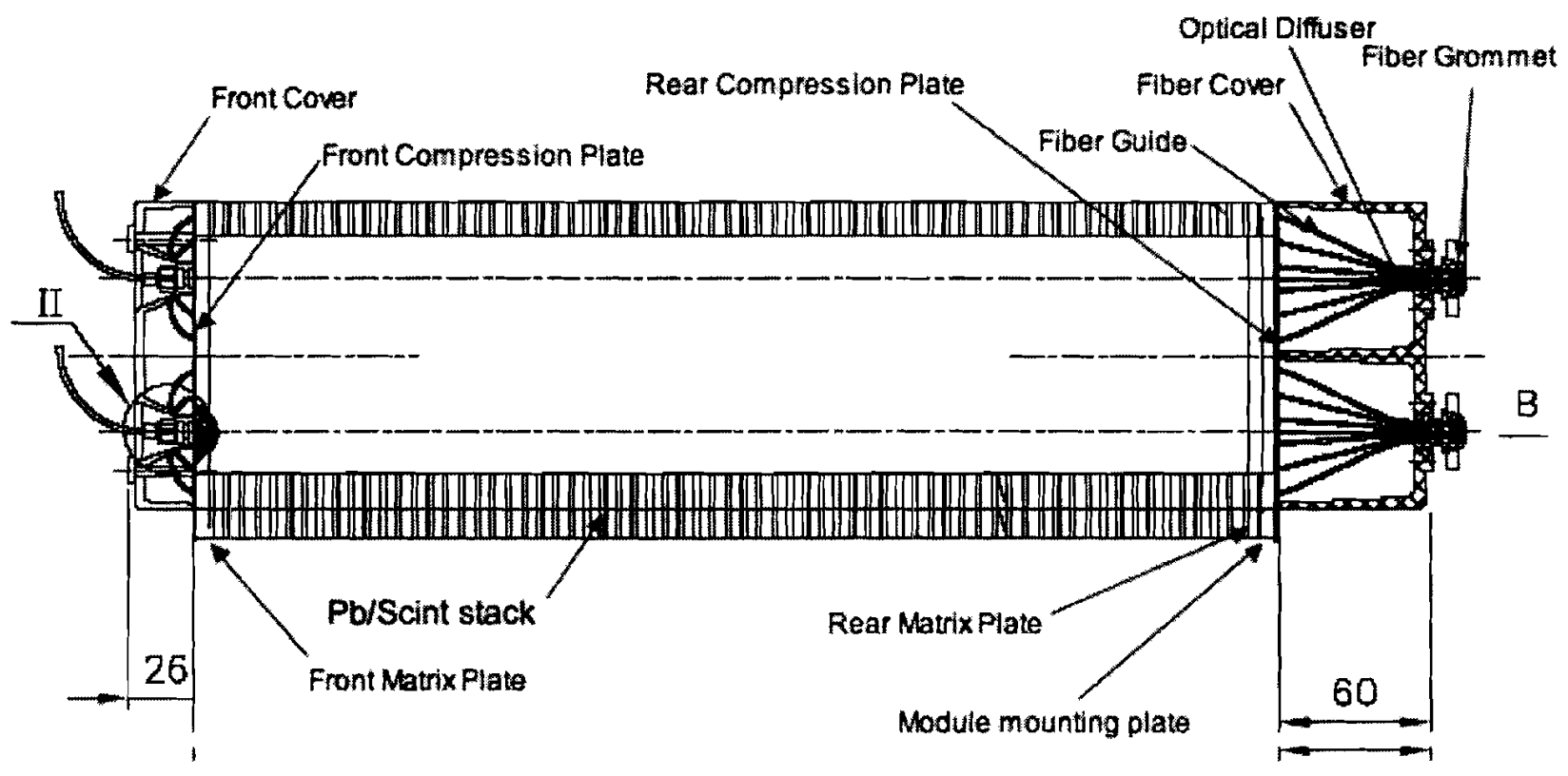

Figure 1. An EMCal module (2x2 readout towers).

The EMCal test beam measurements at FNAL will utilize a stacked $4 \times 4$ array of prototype EMCal modules ( $8 \times 8$ towers). All towers will be instrumented with the same model APD and preamplifier as will be used in the ALICE experiment and all channels will be readout with existing prototype front end electronics intended for use in ALICE. The goals of the test beam measurements are: To investigate the energy resolution, linearity, uniformity, and position resolution, using electron beams; To study the energy dependence of the response to electrons and hadrons to determine the particle identification capabilities of the EMCal by shower shape; And to investigate the timing characteristics of the energy signal for crude time-of-flight measurement ( $\sim 1 \mathrm{~ns})$ for use for anti-neutron rejection. Measurements will be made for comparison with different signal shaping times in the front end electronics.

This is a memorandum of understanding between FNAL and the ALICE EMCal group. This memorandum is intended solely for the purpose of providing a work allocation for FNAL and the ALICE EMCal group. It reflects an arrangement that is currently satisfactory to the parties involved. It is recognized, however, that changing circumstances of the evolving research program will necessitate revisions. The parties agree to negotiate amendments to this memorandum to reflect such revisions. 


\section{PERSONNEL AND INSTITUTIONS:}

Spokesman and physicist in charge of

beam tests:

Terry Awes, Oak Ridge National Laboratory

Fermilab liaison:

Erik Ramberg

The ALICE EMCal group members and others who may participate in the test beam measurements are:

1.1 University of California - Berkeley: H.Crawford, E.Judd

1.2 University of California - Los Angeles: V.Ghazikhanian, H.Huang, S.Trentalage, C.Whitten

1.3 Creighton University: M.Cherney, J.Seger

$1.4 \quad$ University of Houston: A.Lan, A.Mairani, B.Mayes, L.Pinsky

1.5 Lawrence Berkeley National Laboratory: P.Jacobs, M.v.Leeuwen, G.Odyniec, J.Putschke, H.G.Ritter

1.6 Lawrence Livermore National Laboratory: J.Klay, R.Soltz

1.7 Michigan State University: A.Vander Molen, G.Westfall

1.8 Oak Ridge National Laboratory: T.Awes, V.Cianciolo, D.Silvermyr, P.Stankus, G.Young,

1.9 Ohio State University: T.Humanic, I.Kotov, M.Lisa, B.Nilsen, D.Truesdale

1.10 Purdue University: O.Baranikova, A.Hirsch, R.Scharenberg, F.Wang

1.11 University of Tennessee: Y.Efremenko, K.Read, S.Sorensen

1.12 University of Washington: H.Bichsel, J.Cramer

1.13 Wayne State University: T.Cormier, A.Mischke, A.Pavlinov, V.Petrov, C.Pruneau, J.Riso

\section{EXPERIMENTAL AREA, BEAMS AND SCHEDULE CONSIDERATIONS}

$2.1 \quad$ LOCATION

2.1.1 The experiment is to take place in the MTEST beam line and located in the area designated MT6-B3. In addition, the main control room to the west of the MTEST line will be used to house electronics (NIM, CAMAC, VME, oscilloscope, and computer), and to provide work space. 


\subsection{BEAM}

2.2.1 The tests will use slow resonantly-extracted, Main Injector proton beam focused onto the MTest target. The tests require mixed beams with good particle identification (e/pi/p discrimination) over the full range of available momenta (4-66 GeV).

2.2.2 Resolution: A beam momentum selection with a momentum bite of $\Delta \mathrm{p} / \mathrm{p} \sim 2 \%$ or less is highly desirable. Alternatively, an accurate estimate of the momentum resolution of the test beam at each momentum setting would be needed.

2.1.2 Intensity: In the range of $0.2-10 \mathrm{KHz}$ in an area of approximately 1 square $\mathrm{cm}$, with the rates dependent on momentum. With the current beam line design this is expected to require up to $2 \times 10^{12}$ primary protons per spill. The beam needs to be able to be steered into the middle of a 6 in diameter circle.

\subsection{SETUP}

2.3.1 For the test measurements an array of $4 \times 4$ EMCal modules ( $8 \times 8$ towers) will be used. For handling and stacking purposes the modules will be assembled on a strong-back in strip units of four modules, each strip unit weighing approximately 300 pounds with approximate dimensions of $12 \times 50 \times 50 \mathrm{~cm}$. The strip units will be lifted from packing crates at floor level onto the table in the test beam by means of a commercial, manual hydraulic "cherry picker" rated at 1.5 tons. The hydraulic lift will be provided by the EMCal group if the equivalent is not available from Fermilab. The strip units will be stacked and strapped together to form the array of $4 \times 4$ EMCal modules. The modules will be stacked on top of a thin "lazy-susan" bearing system sitting on top of a moveable platform, to allow the EMCal response dependence on the angle of incidence to be investigated. The moveable platform will consist of a heavy duty horizontal slider/table presently available in the test beam area, provided by Fermilab. Vertical motion will be provided by a commercial scissors table (Southworth model LL1.5) with 1500 pound capacity provided by the EMCal group. The required total range of both horizontal and vertical adjustment is approximately $50 \mathrm{~cm}$. The size of the stacked array is $\sim 51 \times 51 \times 50 \mathrm{~cm}$ with a weight of about $1200 \mathrm{lbs}$ ( $-75 \mathrm{lbs} /$ module). The EMCal readout electronics will be attached to the back of the array of modules. An access space of about $\sim 1 \mathrm{~m}$ behind the EMCal array is needed for installation and access to the electronics. With the moveable platform, the EMCal array can be moved out of the beam if necessary to accommodate downstream users.

2.3.2 Use of a beam Cerenkov counter is needed for electron/pion discrimination (and pion/proton discrimination if more than one Cerenkov counter can be used).

2.3.3 A pair of scintillator paddles, one immediately in front of the EMCal and one far upstream will be used for beam definition and timing. A counter to provide $x-y$ position measurement with $\sim 1 \mathrm{~mm}$ position resolution will be located immediately in front of the EMCal.

\subsection{SCHEDULE}

2.4.1 The intention is to install the EMCal prototype in the test beam, complete the measurements, and remove the prototype, in the shortest time period possible. We anticipate that this could optimistically be completed in a time period of $\sim 3$ weeks 
with steady availability and control of the test beam. The preferred time period is November, 2005.

2.4.2 Details of the measurement plan: 2-3 days of physical setup in the test beam. 3-4 days of setup and debugging of readout of EMCal and associated beam trigger counters. Then 1) 1-2 days for gain-matching scan with $\sim 10 \mathrm{GeV} / \mathrm{c}$ electrons through all 64 towers ( 1000 e /tower). 2) 1 day for Momentum scan with electrons to investigate linearity and energy and position resolution. 3) 3 days to repeat 1-2) with alternative electronics shaping time. 3) 2 days for detailed position and angle of incidence scan with $\sim 10 \mathrm{GeV} / \mathrm{c}$ electrons to investigate uniformity of response. 4) 3 days for high statistics momentum/position/angle scans with $\mathrm{e} / \mathrm{pi} / \mathrm{p}$ to investigate particle identification by shower shape. 5) 1 day with thick $C$ target in beam just upstream of the EMCal as neutral pion production source via charge exchange reaction to study energy-position resolution via neutral pion invariant mass reconstruction.

\section{RESPONSIBILITIES BY INSTITUTION - NON FERMILAB}

3.1 The EMCal modules and all of its associated readout electronics will be brought by the ALICE EMCal group.

3.2 The array of EMCal prototype modules will be assembled and disassembled on the moveable platform by members of the EMCal group.

3.3 The DAQ computer will be provided by the EMCal group.

3.4 ALICE test beam users will ensure that safe lifting practices are used in the installation of calorimeter modules, including having a minimum of 2 people involved and making sure that no installer has a pre-existing condition that would preclude them from lifting the required weight.

\section{RESPONSIBILITIES BY INSTITUTION - FERMILAB}

\subsection{Fermilab Accelerator Division:}

4.1.1 Use of MTest beam.

4.1.1 Maintenance of all existing standard beam-line elements such as SWICs, loss monitors, instrumentation, controls, clock distribution and power supplies.

4.1.2 Beam controls console and remote data logging capability (ACNET)

4.1.3 Reasonably rapid access to our equipment in the test beam. Such access is anticipated to be less than once per hour, typically more like once per shift.

4.1.4 Logic signal at experimenter electronics racks that has a constant phase (within 1-2 $\mathrm{ns}$ in a given hour) with respect to the arrival of beam buckets at the test apparatus.

4.1.5 No experiment-owned devices need interfacing to the Accelerator Division control system.

4.1.6 Position and focus of the beam on the experimental devices under test will be under control of MCR. Control of secondary devices that provide these functions may be delegated to the experimenters as long as it does not violate the Shielding Assessment or provide potential for significant equipment damage.

4.1.7 The integrated effect of running this and other SY120 beams will not reduce the antiproton stacking rate or protons on target for the neutrino program by more than 
5\% globally, with the details of scheduling to be worked out between the experimenters and the Office of Program Planning.

\subsection{Fermilab Particle Physics Division}

4.2.1 The test-beam efforts in this MOU will make use of the Meson Test Beam Facility. Requirements for the beam and user facilities are given in Section 2. The Fermilab Particle Physics Division will be responsible for coordinating overall activities in the MTest beam-line, including use of the user beam-line controls, readout of the beamline detectors, and MTest gateway computer.

4.2.2 Maintenance of detector elements in the beamline used to trigger on beam (scintillators, Cerenkov counters, MWPC, etc.)

4.2.3 A horizontal moveable platform with remote control and readout will be provided for mounting the EMCal in the test beam. An engineering assessment of this platform will be done by the PPD. (0.5 person-weeks)

4.2.1 Initial assistance with operation and readout of all beam trigger counters will be provided to the ALICE EMCal group. (0.5 person-weeks)

\subsection{Fermilab Computing Division}

4.3.1 The ALICE EMCal group will supply all needed computer equipment.

4.3.2 FNAL will provide Ethernet access in the counting house with access to a printer.

\subsection{Fermilab ES\&H Section}

4.4.1 We will require assistance with safety reviews.

V. SUMMARY OF COSTS

Source of Funds $[\$ K] \quad$ Equipment Operating Personnel

(person-weeks)

Accelerator Division

Particle Physics Division

Computing Division

$\$ 0 \mathrm{~K}$
0
0

$\$ 0 \mathrm{~K}$

0

0

0

0

1.0

Totals Fermilab

$\$ 0 \mathrm{~K}$

0

1.0 


\section{SPECIAL CONSIDERATIONS}

6.1 The responsibilities of the spokesman of the ALICE EMCal test group and the procedures to be followed by experimenters are found in the Fermilab publication "Procedures for Experimenters" (http://www.fnal.gov/directorate/documents/index.html). The spokesman agrees to those responsibilities and to follow the described procedures.

6.2 To carry out the experiment a number of Environmental, Safety and Health (ES\&H) reviews are necessary. This includes creating a Partial Operational Readiness Clearance document in conjunction with the standing Particle Physics Division committee. The spokesman of the ALICE EMCal test group will follow those procedures in a timely manner, as well as any other requirements put forth by the division's safety officer.

6.3 The spokesman of the ALICE EMCal test group will ensure that at least one person is present at the Meson Test Beam Facility whenever beam is delivered and that this person is knowledgeable about the experiment's hazards.

6.4 All regulations concerning radioactive sources will be followed. No radioactive sources will be carried onto the site or moved without the approval of the Fermilab ES\&H section.

6.5 All items in the Fermilab Policy on Computing will be followed by experimenters. (http://computing.fnal.gov/cd/policy/cpolicy.pdf).

6.6 The spokesman of the ALICE EMCal test group will undertake to ensure that no PREP or computing equipment be transferred from the experiment to another use except with the approval of and through the mechanism provided by the Computing Division management. They also undertake to ensure that no modifications of PREP equipment take place without the knowledge and consent of the Computing Division management.

6.7 The ALICE EMCal test group will be responsible for maintaining and repairing both the electronics and the computing hardware supplied by them for the experiment. Any items for which the experiment requests that Fermilab performs maintenance and repair should appear explicitly in this agreement.

6.8 At the completion of the experiment:

6.8.1 The spokesman of the ALICE EMCal test group is responsible for the return of all PREP equipment, computing equipment and non-PREP data acquisition electronics. If the return is not completed after a period of one year after the end of running the spokesman of the ALICE EMCal test group will be required to furnish, in writing, an explanation for any non-return.

6.8.2 The experimenters agree to remove their experimental equipment as the Laboratory requests them to. They agree to remove it expeditiously and in compliance with all ES\&H requirements, including those related to transportation. All the expenses and personnel for the removal will be borne by the experimenters.

6.8.3 The experimenters will assist the Fermilab Divisions and Sections with the disposition of any articles left in the offices they occupied, including computer printout and magnetic tapes.

6.8.4 An experimenter will report on the test beam effort at a Fermilab All Experimenters Meeting. 
10/17/2005 00:54

3447322

PHENIX *DB *

PAGE _ 02/02

SIGNATURES:

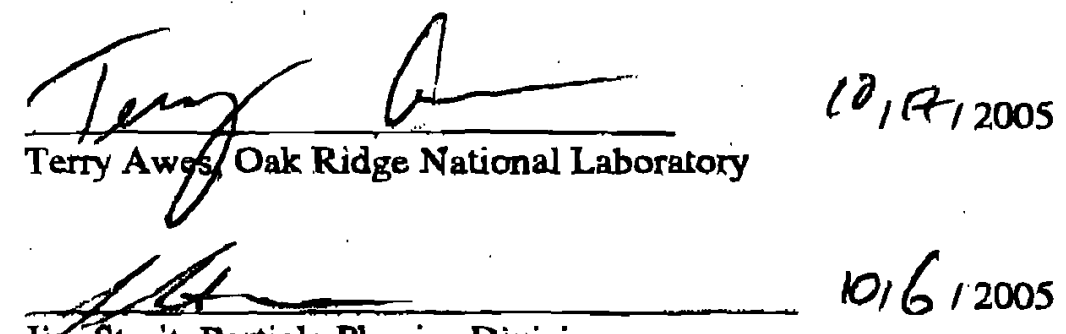

Join Strait, Particle Physics Division
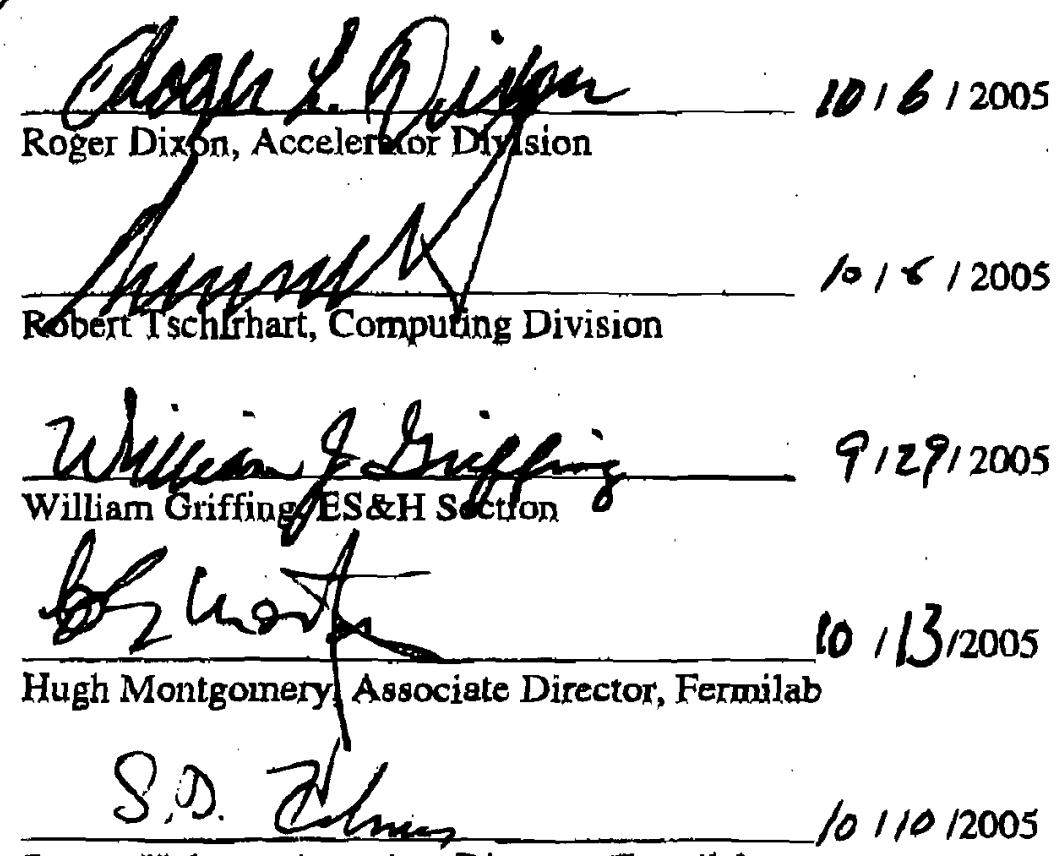

Steven Holmes, Associate Director, Fermilab

10 


\section{APPENDIX I - ALICE ELECTROMAGNETIC CALORIMETER TEST - EQUIPMENT POOL NEEDS}

Equipment Pool items needed for Fermilab test beam. All items related to implementation of trigger logic and readout of beam line counters:

\section{$\underline{\text { Description }}$}

A) NIM crate, with cooling fans, with NIM modules for beam trigger logic.

B) CAMAC crate and power supply, with CAMAC modules for readout of beam counters. 


\section{APPENDIX II - Hazard Identification Checklist}

Items for which there is anticipated need have been checked

\begin{tabular}{|c|c|c|c|c|c|}
\hline \multicolumn{2}{|c|}{ Cryogenics } & \multicolumn{2}{|c|}{ Electrical Equipment } & \multicolumn{2}{|r|}{$\begin{array}{c}\text { Hazardous/Toxic } \\
\text { Materials }\end{array}$} \\
\hline \multicolumn{2}{|c|}{ Beam line magnets } & \multicolumn{2}{|c|}{ Cryo/Electrical devices } & & List hazardous/toxic materials \\
\hline \multicolumn{2}{|c|}{ Analysis magnets } & \multicolumn{2}{|c|}{ capacitor banks } & & $\begin{array}{l}\text { planned for use in a beam line or } \\
\text { experimental enclosure: }\end{array}$ \\
\hline \multicolumn{2}{|c|}{ Target } & \multicolumn{2}{|c|}{$\begin{array}{l}\text { high voltage }(1000 \mathrm{~V} \text { at } 1.00 \mathrm{~A} \\
\max )\end{array}$} & & \\
\hline \multicolumn{2}{|c|}{ Bubble chamber } & \multicolumn{2}{|c|}{ exposed equipment over $50 \mathrm{~V}$} & & \\
\hline \multicolumn{2}{|c|}{ Pressure Vessels } & \multicolumn{2}{|c|}{$\begin{array}{c}\text { Flammable Gases or } \\
\text { Liquids }\end{array}$} & & \\
\hline & inside diameter & \multicolumn{2}{|l|}{ Type: } & & \\
\hline & operating pressure & \multicolumn{2}{|l|}{ Flow rate: } & & \\
\hline & window material & \multicolumn{2}{|l|}{ Capacity: } & & \\
\hline & window thickness & \multicolumn{2}{|c|}{ Radioactive Sources } & & \\
\hline \multicolumn{2}{|c|}{ Vacuum Vessels } & \multicolumn{2}{|c|}{ permanent installation } & \multicolumn{2}{|r|}{ Target Materials } \\
\hline & inside diameter & \multicolumn{2}{|c|}{ temporary use } & & Beryllium (Be) \\
\hline & operating pressure & \multicolumn{2}{|l|}{ Type: } & & Lithium (Li) \\
\hline & window material & \multicolumn{2}{|l|}{ Strength: } & & Mercury $(\mathrm{Hg})$ \\
\hline & window thickness & \multicolumn{2}{|c|}{ Hazardous Chemicals } & & Lead $(\mathrm{Pb})$ \\
\hline \multicolumn{2}{|r|}{ Lasers } & \multicolumn{2}{|c|}{ Cyanide plating materials } & & Tungsten (W) \\
\hline \multicolumn{2}{|c|}{ Permanent installation } & \multicolumn{2}{|c|}{ Scintillation Oil } & & Uranium (U) \\
\hline \multicolumn{2}{|c|}{ Temporary installation } & \multicolumn{2}{|c|}{ PCBs } & $\mathbf{x}$ & Other : Iron (Fe) \\
\hline \multicolumn{2}{|c|}{ Calibration } & \multicolumn{2}{|c|}{ Methane } & \multicolumn{2}{|r|}{ Mechanical Structures } \\
\hline \multicolumn{2}{|c|}{ Alignment } & \multicolumn{2}{|c|}{ TMAE } & $\mathrm{X}$ & Lifting devices \\
\hline type: & & $\mathrm{TE}$ & & $\mathbf{X}$ & Motion controllers \\
\hline Wattage: & & pho & graphic developers & & scaffolding/elevated platforms \\
\hline class: & & Oth & : Activated Water? & & Others \\
\hline
\end{tabular}

